Published in final edited form as:

Magn Reson Med. 2016 June ; 75(6): 2286-2294. doi:10.1002/mrm.25785.

\title{
Improved black-blood imaging using DANTE-SPACE for simultaneous carotid and intracranial vessel wall evaluation
}

\author{
Yibin Xie ${ }^{1,2}$, Qi Yang ${ }^{1,3}$, Guoxi Xie ${ }^{1,4}$, Jianing Pang ${ }^{1}$, Zhaoyang Fan ${ }^{1}$, and Debiao $\mathrm{Li}^{1,2,{ }^{\text {** }}}$ \\ ${ }^{1}$ Biomedical Imaging Research Institute, Cedars-Sinai Medical Center, Los Angeles, CA, USA \\ ${ }^{2}$ Department of Bioengineering, University of California, Los Angeles, CA, USA \\ ${ }^{3}$ Department of Radiology, Xuanwu Hospital, Capital Medical University, Beijing, China \\ ${ }^{4}$ Shenzhen Key Lab for MRI, Shenzhen Institutes of Advanced Technology, Chinese Academy of \\ Sciences, Shenzhen, China
}

\section{Abstract}

Purpose-The purpose of this work is to develop a 3D black-blood imaging method for simultaneously evaluating carotid and intracranial arterial vessel wall with high spatial resolution and excellent blood suppression with and without contrast enhancement.

\begin{abstract}
Methods-DANTE preparation module was incorporated into SPACE sequence to improve blood signal suppression. Simulations and phantom studies were performed to quantify image contrast variations induced by DANTE. DANTE-SPACE, SPACE and 2D TSE were compared for apparent SNR, CNR and morphometric measurements in fourteen healthy subjects. Preliminary clinical validation was performed in six symptomatic patients.
\end{abstract}

Results-Apparent residual luminal blood was observed in 5 (pre-CE) and 9 (post-CE) subjects with SPACE, and only 2 (post-CE) subjects with DANTE-SPACE. DANTE-SPACE showed $31 \%$ (pre-CE) and $100 \%$ (post-CE) improvement in wall-to-blood CNR over SPACE. Vessel wall area measured from SPACE was significantly larger than that from DANTE-SPACE due to possible residual blood signal contamination. In patients DANTE-SPACE showed the potential to detect vessel wall dissection and identify plaque components.

Conclusion-DANTE-SPACE significantly improved arterial and venous blood suppression compared with SPACE. Simultaneous high-resolution carotid and intracranial vessel wall imaging to potentially identify plaque components was feasible with scan time under 6 minutes.

\section{Keywords}

DANTE; SPACE; black-blood imaging; vessel wall imaging; carotid artery imaging; intracranial artery imaging

\footnotetext{
${ }^{* *}$ Corresponding author: Debiao Li, PhD, Address: Cedars-Sinai Medical Center, Biomedical Imaging Research Institute, 8700 Beverly Blvd., PACT Suite 800, Los Angeles, CA, USA 90048, Tel: 424-672-5278, Fax: 310-248-8682, debiao.li@ cshs.org.
} 


\section{Introduction}

Stroke is a global health concern and majority of stroke cases are ischemic stroke. The major underlying cause of ischemic stroke is atherosclerosis. MRI is one of the most promising noninvasive modalities for vessel wall imaging (1). Unlike CT and ultrasound, MRI can provide excellent tissue contrast for directly assessing vessel wall in addition to angiographic images for evaluating luminal stenosis. This advantage became more desirable in light of recent evidences showing that stenosis alone is a poor predictor for cerebral events as significant luminal narrowing may be absent in patients with severe atherosclerosis owing to arterial remodeling $(1,2)$. In addition, direct imaging of the blood vessel wall offers the potential to discriminate between different vascular pathologies with similar luminal defects therefore helping determine the optimal treatment (3).

Atherosclerosis is a systematic disease that usually affects multiple vascular beds and diseases in extracranial and intracranial arteries are both commonly associated with ischemic stroke (4). An ideal imaging protocol therefore should cover all of the frequently affected locations including common carotid artery bifurcation, the siphon, the $1^{\text {st }}$ segment of the middle cerebral artery (M1), $1^{\text {st }}$ and $4^{\text {th }}$ segments of the vertebral artery (V1, V4) and the $1^{\text {st }}$ segment of the basilar artery (BA) (5). The conventional extracranial carotid vessel wall imaging is based on multi-slice 2D TSE (6). However, applying this technique to intracranial vessels may lead to inaccuracy due to their small size and tortuous course (7). Recently 3D variable-flip-angle TSE (SPACE for Siemens, VISTA for Philips, and CUBE for GE) emerged as a promising technique for extracranial carotid (8) and intracranial (9) vessel wall that provides several advantages over its $2 \mathrm{D}$ counterpart: 1) higher resolution especially in the slice direction, potentially isotropic; 2) larger anatomical coverage with coronal acquisition; 3) capability of retrospective reformatting with arbitrary slice orientation and thickness. However current SPACE protocols typically focuses on a single station and using multiple scans to cover all frequently affected vascular locations is time-consuming and infeasible for certain patients.

Successful vessel wall imaging requires complete suppression of luminal blood, i.e. blackblood (BB) imaging, to provide a good vessel wall to lumen contrast for assessing vessel wall pathology (10). Commonly used BB techniques such as double inversion recovery (DIR) (11) are more difficult to work with large FOV 3D acquisitions due to larger outflow volume. Despite inherent black-blood effects by SPACE, residual blood signal is often observed in carotid artery which could be mistaken as plaque. Blood suppression in intracranial vessels may be more challenging due to slower flow. To address these issues, additional BB techniques such as flow-sensitive dephasing (MSDE) have been attempted in SPACE imaging, however, with unwanted T2 signal decay $(8,12)$. The use of gadolinium contrast agent for enhancing plaque components (fibrous cap, inflammation etc.) makes post-contrast blood suppression more difficult due to shortened blood T1. Recently a new BB technique using Delay Alternating with Nutation for Tailored Excitation (DANTE) pulse train has been used in gradient echo sequences providing good suppression of slow flow, less signal loss, and less T2 weighting than MSDE preparation (13). We hypothesize that the combination of slow-flow suppression by DANTE preparation and inherent BB effects by SPACE may be a solution for post-contrast vessel wall imaging. 
Based on the aforementioned considerations, the goal of this study is to develop a timeefficient 3D combined carotid and intracranial vessel wall imaging protocol with high isotropic resolution that: 1) covers all frequently affected vascular locations in a single scan; and 2) suppresses blood effectively both pre- and post-contrast.

\section{Methods}

\section{Theoretical and technical considerations}

SPACE is a variant of 3D TSE that significantly improves k-space sampling efficiency with longer echo train duration and shorter echo spacing. The use of longer echo train duration is enabled by using variable flip angle refocusing pulse train that compensates for T2 decay.

Additionally echo spacing is reduced by the use of nonselective refocusing pulses. Satisfactory T1-weighting (or PD-weighting) can be achieved with carefully designed reordering which enables the central part of k-space to be sampled at the beginning of the echo train.

SPACE has inherent flow suppression properties that are primarily based on two mechanisms: 1) Gradient moments induced intravoxel dephasing. Flowing blood spins within a voxel has a range of velocities and accelerations that will result in a widespread of phase dispersion leading to signal cancellation under the influence of uncompensated gradient moments during each echo train. This is less effective when the flow is not parallel to the readout direction, of which the gradient moments are typically much higher than those in the other two directions (14). 2) Stimulated echo induced intravoxel dephasing. The non-180 variable flip angle refocussing pulses used in SPACE introduce various stimulated echoes that go through different coherence pathways than corresponding spin echoes leading to further dephasing of moving spins.

DANTE suppresses signal of moving spins through a different mechanism than SPACE. DANTE consists of a series of low flip angle nonselective pulses interleaved with gradient pulses with short repetition times which drive static spin systems into a steady-state. Flowing spins cannot establish such steady state due to the spoiling effect caused by the applied gradient and the velocity variations throughout the pulse train. This attenuation effect is insensitive to flow rate as long as it is above $0.1 \mathrm{~cm} / \mathrm{s}$, whereas static tissue signal can be largely preserved by the use of small flip angle pulses (15).

The combination of DANTE and SPACE may be complementary for suppressing flow especially in the context of post-CE imaging: 1) Flow suppression in SPACE is directional as the effect is greatest in the readout direction. Therefore it is often under-utilized due to the fact that readout direction is largely limited by anatomical coverage and the course of blood vessels is naturally tortuous (e.g., carotid and intracranial vessels). DANTE can supplement SPACE by applying gradient pulses in the phase encoding directions where its flow suppression is insufficient. 2) DANTE can help suppress slow flow for which SPACE does not generate enough intravoxel phase dispersion. 3) Post-CE the T1 of blood is much shortened (e.g. <150ms) leading to fast recovery of blood signal. Thus even if DANTE can suppress blood completely, its effect will not sustain throughout the readout period (e.g. 
$>150 \mathrm{~ms})$. SPACE generates intravoxel dephasing throughout its echo train thus can help maintain the black-blood effect.

The sequence diagram of DANTE-SPACE is shown in Figure 1, consisting of a DANTE preparation module, an optional anti-aliasing regional saturation module (R-sat) and a fat saturation module (F-sat) played out immediately before SPACE readout module.

\section{Numerical simulations and phantom study}

Bloch equation simulations were performed to investigate the effects of DANTE module on SPACE images in terms of image contrast. Using the approximation of nonflowing magnetization as described by $\mathrm{Li}$ et al. in (15), a range of $\mathrm{T} 1(400 \sim 1550 \mathrm{~ms})$ and $\mathrm{T} 2$ (20 300ms) values relevant to plaque tissue types were simulated. The relative signal differences between DANTE-SPACE and SPACE were quantified. In addition, contrast ratios of different plaque tissue types including normal wall, recent hemorrhage, lipid core and fibrous tissue were calculated and compared between DANTE-SPACE and SPACE. Reference signal intensity was based on skeletal muscle, a commonly used reference for identifying carotid plaque components (16). Relaxation parameters (T1, T2 in msec) were assumed for normal wall $(1115,54)$, recent hemorrhage $(500,107)$, lipid core $(720,37)$, fibrous tissue $(1006,56)$, fibrous tissue after CE $(600,40)$, and skeletal muscle $(1412,50)$ based on literature values $(17,18)$. Different combinations of DANTE parameters including flip angle, echo train length, and echo spacing were tested. Other imaging parameters were kept identical as the following in vivo studies. Coding and visualization were written in MATLAB (The Mathworks, Inc, Natick, MA; version 2010b).

A phantom was constructed to evaluate the contrast variations. It consists of eight test tubes containing deionized water doped with different concentrations of gadolinium submerged in a cylindrical water tank. It was scanned using DANTE-SPACE, SPACE, and T1w 2D TSE. Different combinations of DANTE parameters including flip angle $\left(5^{\circ}\right.$ to $\left.10^{\circ}\right)$, echo train length (90 to 200), and echo spacing $(0.5 \mathrm{~ms}$ or $1 \mathrm{~ms})$ were tested. The resultant images were normalized and signal intensities were measured from each test tube. In comparison, intraclass correlation coefficient (ICC) and mean square error (MSE) were calculated between DANTE-SPACE and SPACE, as well as between DANTE-SPACE and 2D TSE, respectively.

\section{Healthy subject and patient studies}

Study population-The study was approved by institutional review board. Fourteen healthy volunteers ( 8 males, 6 females; age range $23-60$, mean 37 ) with no history of carotid or intracranial vascular disease and six patients (4 males, 2 females; age range 37-85, mean 60) with known carotid and/or intracranial stenosis based on previous MRA or CTA were consecutively recruited for the study. Informed consent was obtained from all study participants.

System and coil configuration-All studies were performed on a clinical 3T MR system (MAGNETOM Verio; Siemens AG Healthcare, Erlangen, Germany). The scanner's integrated body coil was used for RF transmission. Due to coil availability, a 12-channel 
integrated head/neck coil was used together with a 4-channel bilateral special purpose surface coil on the healthy subjects, whereas the same head/neck coil was used together with a 4-channel phased-array carotid coil (Machinet BV, Elde, The Netherlands) on the patients.

Imaging protocol-Standard triple-plane localizers were followed by a 3D time-of-flight (TOF) MRA for localizing carotid and intracranial arteries. As shown in Figure 2A, TOF covers the vasculature in the entire neck and part of brain up till the level of corpus callosum with multiple transverse slabs. Maximum intensity projection (MIP) images of TOF were then used for the planning of the subsequent vessel wall imaging.

Conventional multi-slice T1w 2D TSE images were acquired as reference. Sixteen slices centered at the carotid bifurcations were prescribed with no gap in-between. To further suppress arterial and venous flow, a pair of parallel saturation bands each with thickness of $50 \mathrm{~mm}$ were placed $10 \mathrm{~mm}$ above and below the slices group, as described in previous studies $(8,19)$. Other imaging parameters include: $F O V=190 \times 190 \mathrm{~mm}^{2} ;$ in-plane resolution $=0.74 \times 0.74 \mathrm{~mm}^{2} ;$ slice thickness $=2 \mathrm{~mm} ; \mathrm{TR} / \mathrm{TE}=770 / 9.4 \mathrm{~ms}$; turbo factor $=7$; pixel bandwidth $=528 \mathrm{~Hz}$; refocussing flip angle $=180^{\circ}$; total scan time $=3^{\prime} 33^{\prime \prime}$.

SPACE and DANTE-SPACE images were acquired afterwards. As shown in Figure 2A, an oblique coronal slab slightly rotated transversely were prescribed to include both carotid and vertibrobasilar arterial systems with a coverage of $190 \mathrm{~mm}$ in the head-feet direction. It consists of 80 slices each with thickness of $0.74 \mathrm{~mm}$, yielding a total slab thickness of $59.2 \mathrm{~mm}$ in the anterior-posterior direction. A variable flip angle scheme was used for refocusing pulses in order to achieve $\mathrm{T} 1$ image contrast. Additional parameters regarding the SPACE readout module include: $\mathrm{FOV}=166 \times 190 \mathrm{~mm}^{2} ;$ spatial resolution $=0.74 \mathrm{~mm}$ isotropic; TR/TE $=770 / 22 \mathrm{~ms}$; turbo factor $=31$; pixel bandwidth $=528 \mathrm{~Hz}$; parallel imaging iPAT factor $=2$; total scan time $=5^{\prime} 57^{\prime \prime}$. After testing on healthy subjects, DANTE echo train parameters were optimized based on the following tradeoffs and constraints: 1) larger flip angle provides higher flow sensitivity however at the price of static tissue SNR and increased SAR; 2) higher number of pulses is desired to reach true steady state of static tissue but leads to prolonged scan. Final parameters choices for DANTE module include: flip angle $=10^{\circ}$, phase increment $=0^{\circ}$, number of pulses $=100$; inter-pulse repeat time $=$ $1 \mathrm{~ms} ; \mathrm{Gxyz}=25 \mathrm{mT} / \mathrm{m}$.

Post-CE imaging was performed 2 minutes after an intravenous injection of gadoversetamide (OptiMARK, Mallinckrodt, Hazelwood, MO, USA) at the dose of 0.1 $\mathrm{mmol} / \mathrm{kg}$ body weight. DANTE-SPACE and SPACE images were acquired in randomized order followed by T1w 2D TSE. All imaging parameters were kept identical to those of preCE imaging.

\section{Image processing and analysis}

Signal, noise and morphometric measurements-Images were reviewed and analyzed by two reviewers including an experienced radiologist on a clinical workstation (Leonardo, Siemens AG Healthcare). Multi-planar reformatting (MPR) was performed on each 3D dataset at internal carotid arteries right above carotid bifurcations where residual blood artifacts were most commonly observed. SPACE and DANTE-SPACE images were 
de-identified and reviewed side-by-side following an automatic co-registration to correct any possible inter-scan motion. Four contiguous 2D cross-sectional images with thickness of $2 \mathrm{~mm}$ (matched with 2D TSE) of carotid arteries were reconstructed from each dataset for the measurements. Arterial lumen signal $\left(\mathrm{S}_{\text {lumen }}\right)$ was measured as the mean signal intensity within a manually drawn region of interest (ROI) covering the entire arterial lumen. When apparent residual arterial blood was observed, signal of blood $\left(\mathrm{S}_{\text {blood }}\right)$ was measured with a ROI covering the residual blood area. Identical blood ROIs were used on DANTE-SPACE and SPACE image in order to avoid potential bias in the comparison. Vessel wall signal $\left(S_{w a l l}\right)$ was measured as the mean signal intensity of a one-pixel-width circumferential path traced through the middle of the arterial wall in order to mitigate potential inaccuracy induced by partial volume effects (20). Similarly, venous blood signal $\left(\mathrm{S}_{\mathrm{venous}}\right)$ were measured by a ROI covering the entire lumen of the internal jugular vein. Cerebrospinal fluid (CSF) signal $\left(\mathrm{S}_{\mathrm{csf}}\right)$ was measured at a homogenous area surrounding the cervical spinal cord. Noise level $(\sigma)$ was defined as the signal standard deviation within a ROI drawn in the adjacent air space of the image uncontaminated by artifacts. Same noise measurement area was used on DANTE-SPACE and SPACE images. The apparent SNR and CNR were defined as:

$$
\begin{aligned}
\mathrm{SNR}_{\mathrm{x}} & =0.695 \times \mathrm{S}_{\mathrm{x}} / \sigma \\
\mathrm{CNR}_{\mathrm{x}-\text { to- } \mathrm{y}} & =0.695 \times\left(\mathrm{S}_{\mathrm{x}}-\mathrm{S}_{\mathrm{y}}\right) / \sigma
\end{aligned}
$$

Where $S_{\mathrm{X}}$ denotes the signal intensity of $\mathrm{x}$ anatomy (arterial lumen, arterial vessel wall, venous lumen, or $\mathrm{CSF}$ ); and $\mathrm{S}_{\mathrm{y}}$ denotes the signal intensity of background (arterial lumen or residual arterial blood). Multiplier 0.695 is the multi-coil correction factor (21). Arterial lumen area and vessel wall area were measured on SPACE, DANTE-SPACE and slicematched 2D TSE images. The inner and outer boundaries of vessel wall were manually traced to form two concentric ROIs. Lumen area was measured by the inner ROI whereas vessel wall area was defined as the area difference between the two ROIs. All measurements were performed blinded to the image type and images from the same subject were analyzed at the same session to ensure consistency.

\section{Statistical analysis}

R statistical programming language (ver. 3.0.3, The R Foundation for Statistical Computing, Vienna, Austria) was used for all statistical analysis. A two-tailed paired Student's t-test was performed between DANTE-SPACE and SPACE images for $\mathrm{SNR}_{\text {lumen }}, \mathrm{SNR}_{\text {wall }}$, $\mathrm{CNR}_{\text {wall-to-lumen }}, \mathrm{CNR}_{\text {wall-to-blood }}, \mathrm{LA}$, and WA following verification of data normality with Shapiro-Wilk test. Additionally, agreement in WA and LA measurements between DANTESPACE and 2D TSE were assessed with intraclass correlation coefficient. All numerical data are presented in the format of mean $\pm \mathrm{SD}$ and statistical difference is considered significant when $\mathrm{p}<0.05$. 


\section{Results}

Figure 2B and Figure 2C are representative curved MPRs of pre- and post-CE DANTESPACE images from a healthy subject showing integrated views of carotid arteries, middle cerebral arteries, vertebral arteries, basilar arteries and major braches up to anterior/posterior cerebral arteries.

Figure 3A shows an example of the simulation results for the percentage relative signal differences between DANTE-SPACE and SPACE within the T1 range of 400 1550ms and T2 range of 20 300ms. It is important to note that simulation results will vary based on DANTE parameters especially DANTE flip angle and in this particular example DANTE and SPACE parameters were assumed identical to the in vivo studies. Signal differences between the two had little dependence on T1 values and showed larger variations towards the lower T2 range ( $<30 \mathrm{~ms})$, although all of which were below $10 \%$ across the simulated ranges.

Figure 3B shows the comparison between DANTE-SPACE and SPACE for image contrast ratios that are relevant to the identification of different plaque components. The differences between the two sequences were below $3 \%$ for all components.

The phantom results in Figure 3C verified that the addition of DANTE to the SPACE sequence had little impact on the resultant images in terms of contrast variations. The ICC between the signal intensities of DANTE-SPACE and SPACE was very high, ranging from 0.9989 to 0.9999 depending on different DANTE parameter settings. The MSE between the two was all below 0.0005. Similarly, DANTE-SPACE demonstrated consistent contrast behavior compared with conventional T1w 2D TSE, with the ICC between the two ranging from 0.9595 to 0.9738 , and MSE below 0.009 .

Improved arterial blood suppression by DANTE-SPACE was observed in the in vivo study both pre-CE and post-CE. In the 14 healthy subjects who completed the comparative study between DANTE-SPACE and SPACE, apparent arterial residual blood was observed in 5 (pre-CE) and 9 (post-CE) subjects on SPACE images, and 2 (post-CE) subjects on DANTESPACE images. Figure 4A and Figure 4B are representative examples of improved arterial blood suppression by DANTE-SPACE from two subjects at the carotid bifurcation and the petrous segment of internal carotid, respectively. SPACE images had significant residual blood in the lumen that obscured the vessel wall boundary whereas DANTE-SPACE images had clean lumen free of residual blood with well delineated vessel wall. Quantitative analysis revealed that both pre- and post-CE $\mathrm{SNR}_{\text {lumen }}$ was significantly decreased by DANTE-SPACE compared with SPACE (Table 1). A slight reduction in $\mathrm{SNR}_{\text {wall }}$ with

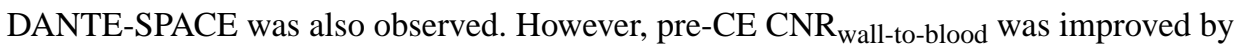
approximately $30 \%$ with DANTE-SPACE $(11.3 \pm 3.6)$ over SPACE $(8.7 \pm 3.6)$ as residual blood was present in the latter. The improvement was more conspicuous for post-CE as $\mathrm{CNR}_{\text {wall-to-blood }}$ was improved by approximately $100 \%$ with DANTE-SPACE (24.0 \pm 7.7$)$ over SPACE (12.0 \pm 4.2$)$.

DANTE-SPACE also enhanced the suppression of venous blood and CSF. Figure 5 shows a representative example of improved venous blood suppression. Post-CE SPACE images had 
largely unsuppressed venous blood causing overlaying flow artifacts along the PE direction (left to right), whereas slice-matched DANTE-SPACE images showed dark venous lumen with well-suppressed venous blood. The level of flow artifacts was also markedly reduced in the DANTE-SPACE images. Quantitatively, the overall $\mathrm{SNR}_{\text {venous }}$ was significantly reduced by DANTE-SPACE compared with SPACE, both pre-CE and post-CE (Table 1). Figure 6 includes a representative case of enhanced CSF suppression by DANTE-SPACE. CSF appeared much darker in the DANTE-SPACE images and the outer boundaries of basilar arteries and its branches were better delineated. In all subjects an approximately $40 \%$ reduction on average was observed in SNR $_{\text {csf }}$ with DANTE-SPACE compared with SPACE (Table 1).

Preliminary clinical study suggested that the proposed DANTE-SPACE protocol had the advantages of a large anatomical coverage and the potential to identify plaque components. Figure 7 shows a case of a patient suspected of carotid artery dissection. The large FOV visualized the carotid arterial system from both sides in its entirety and helped identify the diffused wall thickening along the middle segment of ICA. A thrombus near the bifurcation was also clearly visualized. In-plane and cross-sectional MPR images from the same dataset demonstrated double lumen sign in the intracranial segments of ICA (Figure 7B). Figure 8 shows a case of a patient with recent stoke. Plaque components including intraplaque hemorrhage (IPH), calcification, and thin fibrous cap were clearly visualized in the DANTESPACE images with slice-matched T1w 2D TSE as reference.

Morphometric analysis of the images revealed certain bias between DANTE-SPACE and SPACE images. Increased wall area (WA) and decreased lumen area (LA) were observed in SPACE images compared with DANTE-SPACE images both pre- and post-CE (Table 1). Notably, WA and LA measurements showed significant difference between pre-CE and postCE in SPACE images ( $p<0.001$ ), whereas no such phenomenon was observed in DANTESPACE images ( $p>0.1$ ). DANTE-SPACE and 2D TSE images showed good correlation: the ICC between the two on lumen area was 0.997 for pre-CE and 0.987 for post-CE, whereas the ICC on wall area was 0.996 for pre-CE and 0.989 for post-CE (all $\mathrm{p}<0.001$ ).

\section{Discussion}

In this work we introduced an improved black-blood imaging protocol with DANTE-SPACE for combined evaluation of both carotid and intracranial vessel wall. Superior suppression of arterial blood, venous blood and CSF was observed with DANTE-SPACE compared with SPACE. The proposed protocol covered a large anatomical region with common sites of atherosclerosis formation and 3D imaging with high isotropic resolution $(0.7 \mathrm{~mm})$ was achieved in a clinically acceptable scan time ( $<6 \mathrm{mins})$.

Complete blood suppression is crucial to accurate measurements and evaluation of arterial vessel wall and plaques. However we observed in this study that relying on the native blackblood effects by SPACE alone was not sufficient for many subjects, leaving residual luminal blood that can be mistaken as thickened wall. This was particularly problematic for post-CE imaging as the majority of the subjects in the study showed apparent residual blood in SPACE images, presumably due to vastly shortened blood T1. DANTE-SPACE reduced the 
residual blood signal in these subjects. Notably, $\mathrm{CNR}_{\text {wall-to-blood }}$ was increased by about $30 \%$ and $100 \%$ in DANTE-SPACE images pre-CE and post-CE, respectively. Increased lumen area and reduced vessel wall area were also observed in DANTE-SPACE images which suggested that near-boundary blood signal was further suppressed.

In SPACE images, residual blood artifacts were more likely to occur at certain anatomical locations, including carotid bifurcations, petrous and lacerum segments of ICAs. The irregular anatomy and flow patterns at these locations could result in slow or stagnant flow (8), leading to insufficient phase dispersion during SPACE's echo train. We hypothesize that the improvements by DANTE-SPACE arose from a two-fold mechanism: 1) it suppressed slow flow because of the application of a steady-state pulse train; 2) it supplemented SPACE in directions where native blood suppression was insufficient. In particular, we observed that DANTE-SPACE provided very robust arterial blood suppression in post-CE imaging regardless of the delay time after contrast bolus injection as similar results were achieved even without any delay time (data not shown). This may provide more flexibility in its clinical usage and reduce the possibility of inter-scan motion between pre- and post-CE images. Combining with more advanced imaging acceleration techniques, DANTE-SPACE may be used for 3D black-blood dynamic contrast-enhanced (DCE) imaging for assessing plaque inflammation.

In addition to improved arterial wall imaging, an interesting 'byproduct' of DANTE-SPACE sequence was the further suppression of venous blood and CSF. Venous blood flows at a much lower rate than arterial blood and is not as rhythmic (22). Incomplete and inconsistent venous blood signal intensity between shots could lead to flow artifacts and degradation of image quality. Flow artifacts were generally negligible in pre-CE SPACE images but became more noticeable post-CE imaging. The improved venous blood suppression by DANTESPACE reduced flow artifacts and may also benefit certain applications such as venous thrombus imaging (23). We observed that DANTE-SPACE significantly reduced CSF signal and improved the visualization of vessel walls surrounded by CSF, such as BA and intracranial segments of carotid artery. The improvement in contrast between vessel wall and CSF would become more beneficial for T2- and proton-weighted imaging as CSF naturally appears bright in these images. We hypothesize that the primary reason behind these findings is the property of DANTE module to suppress 'slow' flow. The study by Li et al (15) found that DANTE preparation can attenuate signal from moving spins with velocity as low as $1 \mathrm{~mm} / \mathrm{s}$. However, it is important to note that venous and CSF flow rate may vary greatly depending on locations and disease states. Therefore a systematic study, preferably on patients, is mandatory to further validate these benefits.

The proposed DANTE-SPACE protocol had several advantages compared with the conventional 2D TSE protocol as demonstrated in the preliminary patient study. First, it provided sub-millimeter isotropic spatial resolution $\left(0.7 \times 0.7 \times 0.7 \mathrm{~mm}^{3}\right)$, with about threefold improvement in the slice direction compared with the $2 \mathrm{D}$ technique $\left(0.7 \times 0.7 \times 2.0 \mathrm{~mm}^{3}\right)$.

This may provide substantial clinical benefits for visualizing individual plaque components as their size are typically sub-millimeter (1), smaller than a single voxel in the $2 \mathrm{D}$ technique. We found that the resolution improvement was particularly useful for fibrous cap assessment, which can be very difficult due to partial volume effect in 2D images (24). 
Second, 3D image volume can be easily reformatted to images with arbitrary slice thickness and orientation, providing great flexibility for clinicians to accurately evaluate the extent of lesion. It should also make it easier to correct for misregistration between different scans, for example, in comparing images before and after therapy. Third, the protocol provides large anatomical coverage which enables an integral assessment of both extra- and intracranial vessel wall. Typical 2D TSE protocols use multiple transverse slices that only covers $50 \mathrm{~mm}$ or less of vasculature at a time. Intracranial arterial wall can only be imaged separately and the locations of plaques have to be carefully identified on MRA images before slices can be prescribed at the lesions (25). The proposed protocol covered 190mm of vasculature and can be prescribed without a priori knowledge of plaque locations. The large FOV could also facilitate the evaluation of conditions that affect extensive vascular beds, as shown in the ICA dissection case in the patient study. In certain patients the proposed imaging orientation may cause aliasing from the shoulders, which can be largely eliminated by applying regional saturation bands (Supporting Figure S1).

This study has certain limitations. First, DANTE-SPACE reduced $\mathrm{SNR}_{\text {wall }}$ for approximately $13 \%$ compared with SPACE in this study, resulting in appreciable differences between the two. However, the SNR reduction was less than those typically seen in studies using MSDE, which ranges from $20 \%$ to $40 \%(8,12,20,26)$. Similar SNR advantages by DANTE were also observed by Li et al when using it with FLASH readout (DASH) (13). The DANTE pulse train parameters should be designed according to imaging strategy. Generally the BB effect of DANTE module increases with larger flip angle, at the expense of higher signal loss to static tissue. We used a moderate flip angle of $10^{\circ}$ in the study design as we intended to use DANTE as a supplement to SPACE's native BB effect with less of a penalty on SNR. Second, both the BB module and readout module were relatively long in DANTE-SPACE, which may potentially increase motion sensitivity to the $3 \mathrm{D}$ acquisition. One way to mitigate pulsatile motion is the use of ECG triggering, which should not increase scan time because the TR in the current protocol is similar to a typical RR interval. Navigator (27) or selfgating (28) methods may be adopted in the future. Third, we only designed a T1w protocol in order to reduce the overall exam duration. Multiple contrasts may facilitate the identification of plaque composition (1). It is important to note that T2- and PD-weighted contrasts are also possible to achieve since image contrast is dominated by the readout contrast in DANTE prepared sequences (13).

\section{Conclusions}

DANTE-SPACE is a promising black-blood technique that significantly improves arterial and venous blood suppression pre- and post-CE compared with conventional SPACE technique. Simultaneous high-resolution 3D carotid and intracranial imaging using DANTESPACE is feasible and time-efficient, with the potential ability to identify different plaque components and evaluate plaque vulnerability in the head and neck.

\section{Supplementary Material}

Refer to Web version on PubMed Central for supplementary material. 


\section{Acknowledgments}

This work was supported in part by NIH/NHLBI (R01HL096119) and NSFC (81229001, 81322022). We thank Jaime Shaw for her assistance with the phantom work. We thank Laura Smith, Ed Gill for their technical support and Christy Woo for her assistance with the recruitment of study subjects.

\section{References}

1. Yuan C, Oikawa M, Miller Z, Hatsukami T. MRI of carotid atherosclerosis. Journal of nuclear cardiology : official publication of the American Society of Nuclear Cardiology. 2008; 15:266-275. [PubMed: 18371599]

2. Wasserman BA, Wityk RJ, Trout HH 3rd, Virmani R. Low-grade carotid stenosis: looking beyond the lumen with MRI. Stroke; a journal of cerebral circulation. 2005; 36:2504-2513.

3. Virmani R, Ladich ER, Burke AP, Kolodgie FD. Histopathology of carotid atherosclerotic disease. Neurosurgery. 2006; 59:S219-227. discussion S213-213. [PubMed: 17053606]

4. Adams HP Jr, Bendixen BH, Kappelle LJ, Biller J, Love BB, Gordon DL, Marsh EE 3rd. Classification of subtype of acute ischemic stroke. Definitions for use in a multicenter clinical trial. TOAST Trial of Org 10172 in Acute Stroke Treatment. Stroke; a journal of cerebral circulation. 1993; 24:35-41.

5. Mohr JP, Albers GW, Amarenco P, Babikian VL, Biller J, Brey RL, Coull B, Easton JD, Gomez CR, Helgason CM, Kase CS, Pullicino PM, Turpie AG. American Heart Association Prevention Conference. IV. Prevention and Rehabilitation of Stroke. Etiology of stroke. Stroke; a journal of cerebral circulation. 1997; 28:1501-1506.

6. Cai JM, Hatsukami TS, Ferguson MS, Small R, Polissar NL, Yuan C. Classification of human carotid atherosclerotic lesions with in vivo multicontrast magnetic resonance imaging. Circulation. 2002; 106:1368-1373. [PubMed: 12221054]

7. Antiga L, Wasserman BA, Steinman DA. On the overestimation of early wall thickening at the carotid bulb by black blood MRI, with implications for coronary and vulnerable plaque imaging. Magnetic resonance in medicine : official journal of the Society of Magnetic Resonance in Medicine / Society of Magnetic Resonance in Medicine. 2008; 60:1020-1028.

8. Fan Z, Zhang Z, Chung YC, Weale P, Zuehlsdorff S, Carr J, Li D. Carotid arterial wall MRI at 3T using 3D variable-flip-angle turbo spin-echo (TSE) with flow-sensitive dephasing (FSD). Journal of magnetic resonance imaging : JMRI. 2010; 31:645-654. [PubMed: 20187208]

9. Qiao Y, Steinman DA, Qin Q, Etesami M, Schar M, Astor BC, Wasserman BA. Intracranial arterial wall imaging using three-dimensional high isotropic resolution black blood MRI at 3. 0 Tesla. Journal of magnetic resonance imaging : JMRI. 2011; 34:22-30. [PubMed: 21698704]

10. Dieleman N, van der Kolk AG, Zwanenburg JJ, Harteveld AA, Biessels GJ, Luijten PR, Hendrikse J. Imaging intracranial vessel wall pathology with magnetic resonance imaging: current prospects and future directions. Circulation. 2014; 130:192-201. [PubMed: 25001624]

11. Balu N, Chu B, Hatsukami TS, Yuan C, Yarnykh VL. Comparison between 2D and 3D highresolution black-blood techniques for carotid artery wall imaging in clinically significant atherosclerosis. Journal of magnetic resonance imaging : JMRI. 2008; 27:918-924. [PubMed: 18383253]

12. Zhu C, Graves MJ, Yuan J, Sadat U, Gillard JH, Patterson AJ. Optimization of Improved Motionsensitized Driven-equilibrium (iMSDE) blood suppression for carotid artery wall imaging. Journal of cardiovascular magnetic resonance : official journal of the Society for Cardiovascular Magnetic Resonance. 2014; 16:61. [PubMed: 25160911]

13. Li L, Chai JT, Biasiolli L, Robson MD, Choudhury RP, Handa AI, Near J, Jezzard P. Black-Blood Multicontrast Imaging of Carotid Arteries with DANTE-prepared 2D and 3D MR Imaging. Radiology. 2014:131717.

14. Jara H, Yu BC, Caruthers SD, Melhem ER, Yucel EK. Voxel sensitivity function description of flow-induced signal loss in MR imaging: implications for black-blood MR angiography with turbo spin-echo sequences. Magnetic resonance in medicine : official journal of the Society of Magnetic Resonance in Medicine / Society of Magnetic . Resonance in Medicine. 1999; 41:575-590. 
15. Li L, Miller KL, Jezzard P. DANTE-prepared pulse trains: a novel approach to motion-sensitized and motion-suppressed quantitative magnetic resonance imaging. Magnetic resonance in medicine : official journal of the Society of Magnetic Resonance in Medicine / Society of Magnetic . Resonance in Medicine. 2012; 68:1423-1438.

16. Yuan C, Mitsumori LM, Ferguson MS, Polissar NL, Echelard D, Ortiz G, Small R, Davies JW, Kerwin WS, Hatsukami TS. In vivo accuracy of multispectral magnetic resonance imaging for identifying lipid-rich necrotic cores and intraplaque hemorrhage in advanced human carotid plaques. Circulation. 2001; 104:2051-2056. [PubMed: 11673345]

17. Biasiolli L, Lindsay AC, Chai JT, Choudhury RP, Robson MD. In-vivo quantitative T2 mapping of carotid arteries in atherosclerotic patients: segmentation and T2 measurement of plaque components. Journal of cardiovascular magnetic resonance : official journal of the Society for Cardiovascular Magnetic Resonance. 2013; 15:69. [PubMed: 23953780]

18. Zhu DC, Ferguson MS, DeMarco JK. An optimized 3D inversion recovery prepared fast spoiled gradient recalled sequence for carotid plaque hemorrhage imaging at 3. $0 \mathrm{~T}$. Magnetic resonance imaging. 2008; 26:1360-1366. [PubMed: 18583079]

19. Felmlee JP, Ehman RL. Spatial presaturation: a method for suppressing flow artifacts and improving depiction of vascular anatomy in MR imaging. Radiology. 1987; 164:559-564. [PubMed: 3602402]

20. Koktzoglou I, Li D. Diffusion-prepared segmented steady-state free precession: Application to 3D black-blood cardiovascular magnetic resonance of the thoracic aorta and carotid artery walls. Journal of cardiovascular magnetic resonance : official journal of the Society for Cardiovascular Magnetic Resonance. 2007; 9:33-42. [PubMed: 17178678]

21. Constantinides CD, Atalar E, McVeigh ER. Signal-to-noise measurements in magnitude images from NMR phased arrays. Magnetic resonance in medicine : official journal of the Society of Magnetic Resonance in Medicine / Society of Magnetic . Resonance in Medicine. 1997; 38:852857.

22. Pelc LR, Pelc NJ, Rayhill SC, Castro LJ, Glover GH, Herfkens RJ, Miller DC, Jeffrey RB. Arterial and venous blood flow: noninvasive quantitation with MR imaging. Radiology. 1992; 185:809812. [PubMed: 1438767]

23. Dormont D, Anxionnat R, Evrard S, Louaille C, Chiras J, Marsault C. MRI in cerebral venous thrombosis. J Neuroradiol. 1994; 21:81-99. [PubMed: 8014661]

24. Wasserman BA, Smith WI, Trout HH 3rd, Cannon RO 3rd, Balaban RS, Arai AE. Carotid artery atherosclerosis: in vivo morphologic characterization with gadolinium-enhanced double-oblique MR imaging initial results. Radiology. 2002; 223:566-573. [PubMed: 11997569]

25. Swartz RH, Bhuta SS, Farb RI, Agid R, Willinsky RA, Terbrugge KG, Butany J, Wasserman BA, Johnstone DM, Silver FL, Mikulis DJ. Intracranial arterial wall imaging using high-resolution 3tesla contrast-enhanced MRI. Neurology. 2009; 72:627-634. [PubMed: 19221296]

26. Wang J, Yarnykh VL, Yuan C. Enhanced image quality in black-blood MRI using the improved motion-sensitized driven-equilibrium (iMSDE) sequence. Journal of magnetic resonance imaging : JMRI. 2010; 31:1256-1263. [PubMed: 20432365]

27. Crowe LA, Keegan J, Gatehouse PD, Mohiaddin RH, Varghese A, Symmonds K, Cannell TM, Yang GZ, Firmin DN. 3D volume-selective turbo spin echo for carotid artery wall imaging with navigator detection of swallowing. Journal of magnetic resonance imaging : JMRI. 2005; 22:583588. [PubMed: 16161101]

28. Fan Z, Zuehlsdorff S, Liu X, Li D. Prospective self-gating for swallowing motion: a feasibility study in carotid artery wall MRI using three-dimensional variable-flip-angle turbo spin-echo. Magnetic resonance in medicine : official journal of the Society of Magnetic Resonance in Medicine / Society of Magnetic . Resonance in Medicine. 2012; 67:490-498. 


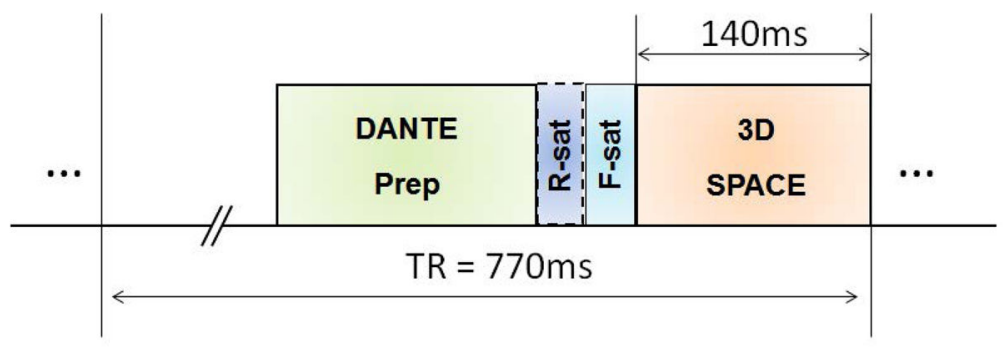

Figure 1.

Sequence diagram of DANTE-SPACE. A DANTE preparation module, an optional antialiasing regional saturation module (R-sat) and a fat saturation module (F-sat) are played out immediately before SPACE readout module. DANTE preparation module consists of a series of small flip-angle RF pulses interleaved with dephasing gradients as described by $\mathrm{Li}$ et al (15). DANTE module is played out immediately before F-sat when R-sat is not needed.

DANTE parameters include: flip angle $=10^{\circ}$, phase increment $=0^{\circ}$, number of pulses $=100$; inter-pulse repeat time $=1 \mathrm{~ms} ; \mathrm{Gxyz}=25 \mathrm{mT} / \mathrm{m}$. 

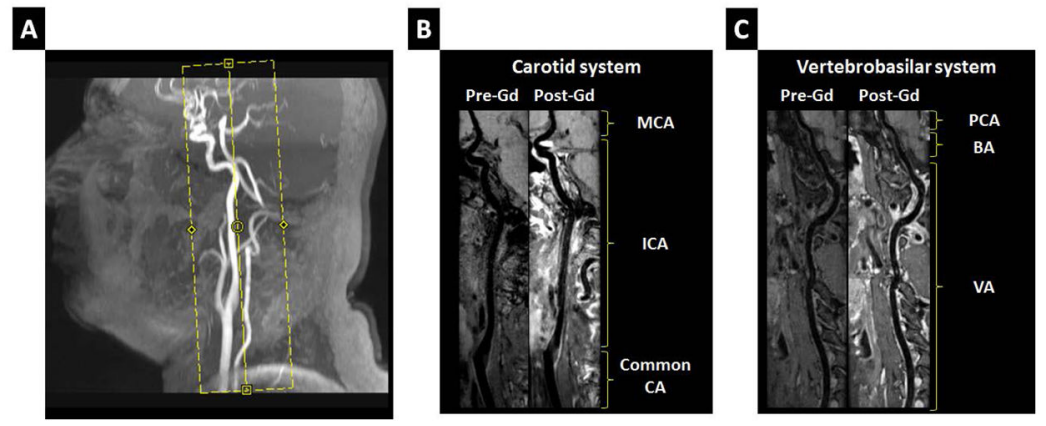

Figure 2.

Combined imaging of carotid and intracranial vessel wall with DANTE-SPACE. (A) Anatomical coverage of the proposed protocol demonstrated on a sagittal MIP image of TOF MRA. A coronal imaging slab was rotated slightly (yellow box) to cover both carotid and vertibrobasilar arterial systems. (B) Reformatted pre- and post-CE DANTE-SPACE images of carotid arteries and major branches from 2 inches below the bifurcation up to the $2^{\text {nd }}$ segment of middle cerebral artery. (C) Reformatted pre- and post-CE DANTE-SPACE images of vertebral arteries, basilar arteries and major braches up to anterior/posterior cerebral arteries. 

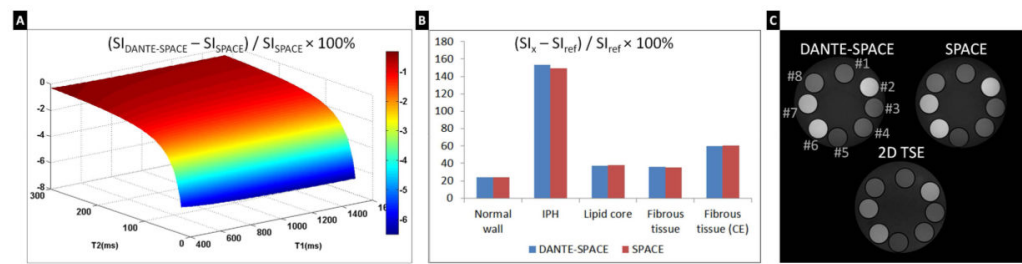

Figure 3.

(A) Simulated results for the percentage relative signal differences between DANTE-SPACE and SPACE across a range of physiologically relevant T1 (400 1550ms) and T2 (20 300ms) values. DANTE-SPACE parameters included: echo train length $=100$; echo spacing $=1 \mathrm{~ms}$; $\mathrm{TR} / \mathrm{TE}=770 / 22 \mathrm{~ms}$. SI = signal intensity. (B) Comparison between DANTE-SPACE and SPACE for image contrast ratios of normal wall, recent hemorrhage, lipid core, and fibrous tissue (each versus skeletal muscle as reference). (C) Images of a T1 phantom consisting of multiple water tubes with different Gd concentration. T1/T2 values of the tubes measured by a basic spin-echo sequence are (in msec): \#1:1276/885; \#2:261/200; \#3:1038/674; \#4:1223/813; \#5:1845/898; \#6:368/291; \#7:428/339, and \#8:1144/757, respectively. Images were acquired by DANTE-SPACE, SPACE and 2D TSE with the same imaging parameters as the in vivo studies. 


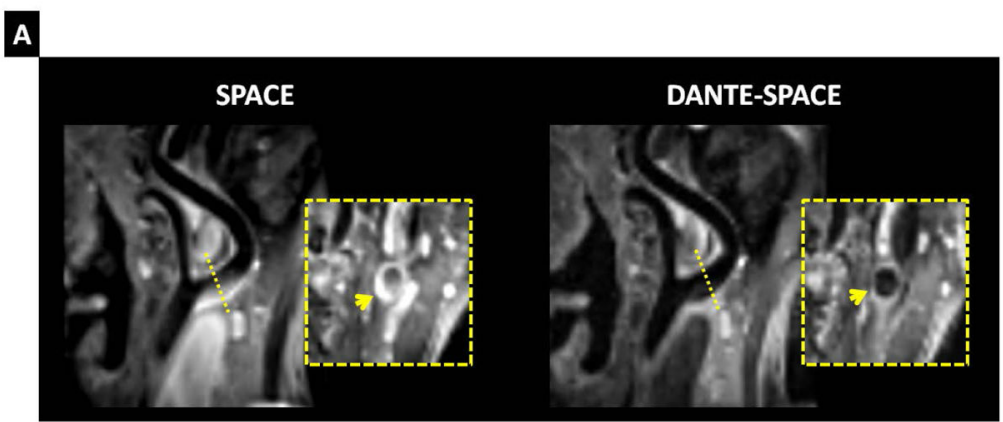

B

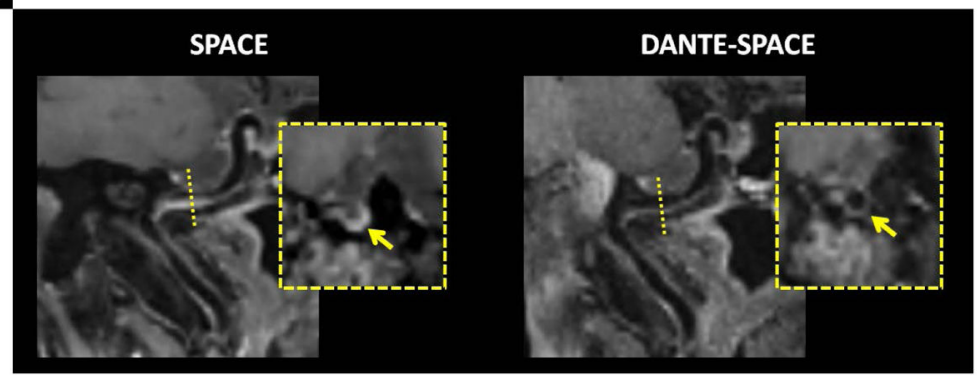

Figure 4.

Two representative examples of improved arterial blood suppression by DANTE-SPACE. (A) Reformatted post-CE images at the carotid bifurcation from a healthy subject acquired using both SPACE and DANTE-SPACE. On the SPACE images significant residual blood was observed at the origin of internal carotid artery. It obscured the boundary between lumen and vessel wall and may be misidentified as a plaque (yellow arrow). On the DANTESPACE images lumen was clean and vessel wall appeared thin. (B) Reformatted post-CE images at internal carotid artery from a symptomatic patient suspected of stroke. Similar findings were observed in comparing SPACE and DANTE-SPACE. 
A

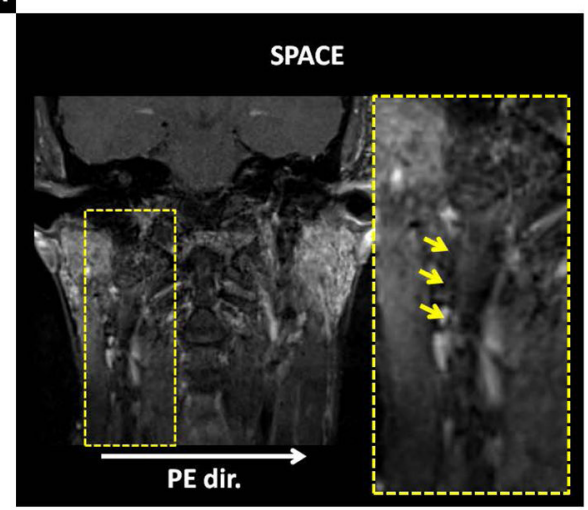

B

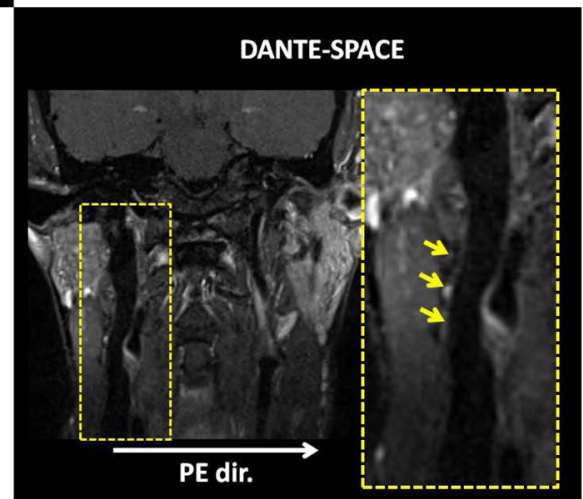

Figure 5.

A representative example of improved venous blood suppression by DANTE-SPACE. (A) On the post-CE SPACE images the venous blood was largely not suppressed causing visible flow artifacts along the PE direction (left to right). (B) On the post-CE DANTE-SPACE images the venous blood was well suppressed and the venous lumen appeared dark (arrows). Also note the reduced level of flow artifacts compared to SPACE images. 


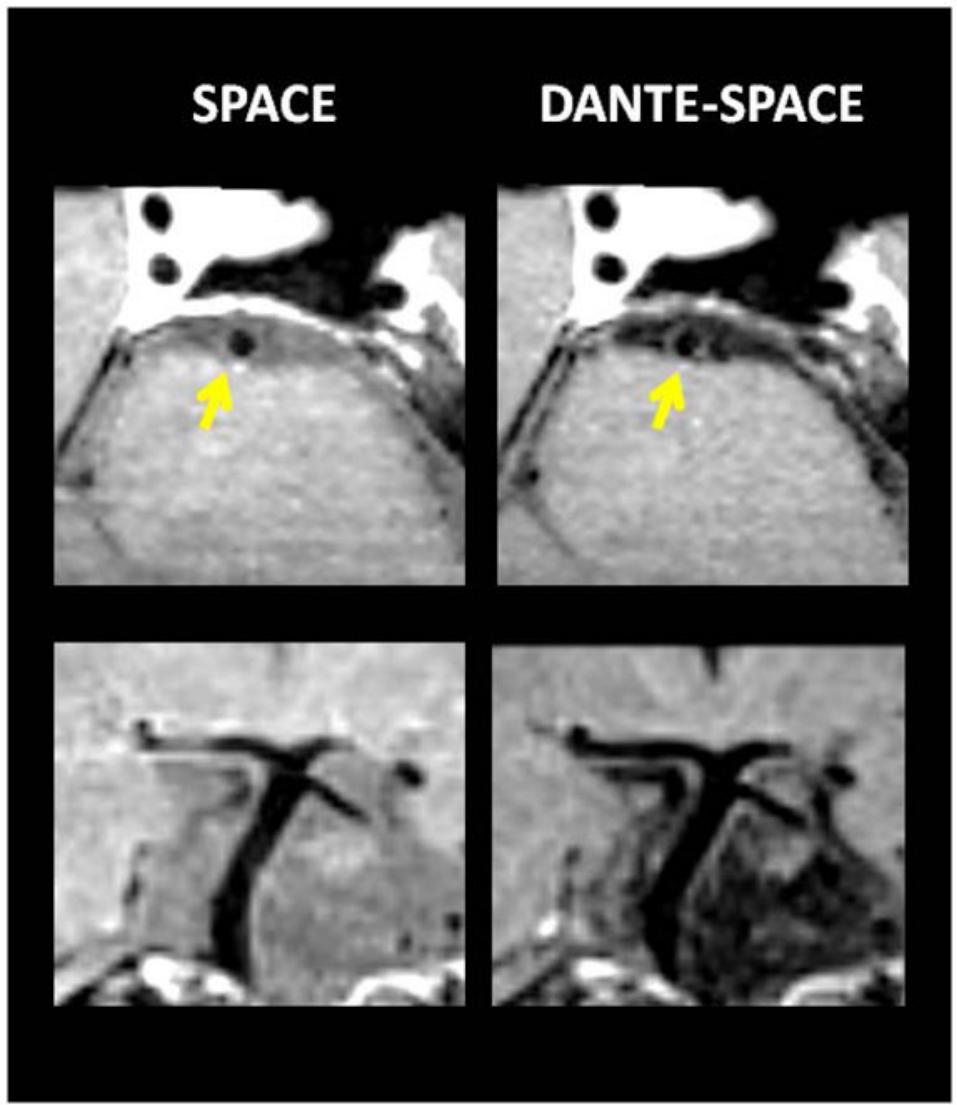

Figure 6.

A representative example of improved CSF suppression by DANTE-SPACE from a healthy subject. The CSF surrounding the basilar arteries and its branches was further suppressed by DANTE-SPACE compared with SPACE, which helped better visualize the outer vessel boundaries. 
A

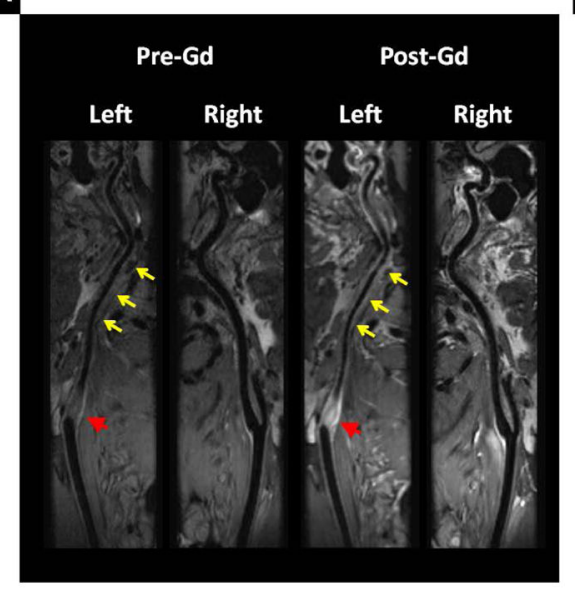

B

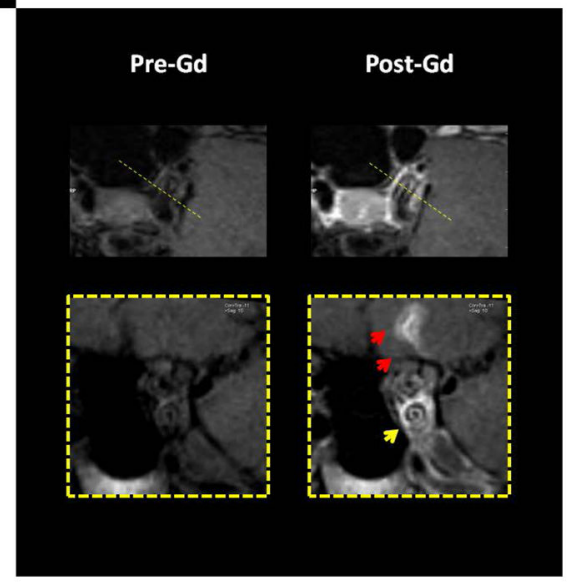

Figure 7.

Reformatted pre- and post-CE DANTE-SPACE images from a patient suspected of internal carotid artery dissection. The patient was a 43 y/o male with stroke symptoms. (A) Curved MPR images of both the dissected carotid arterial wall (left) and the normal carotid arterial wall (right). Note the thrombus near the bifurcation of the left ICA (single red arrow), and diffused arterial wall thickening along the middle segment (triple yellow arrows). (B) Inplane and cross-sectional images showing double lumen sign (single yellow arrow) of intracranial carotid artery which confirmed the diagnosis of dissection. Also note the enhancement of brain infarct in the post-CE image (double red arrows). 

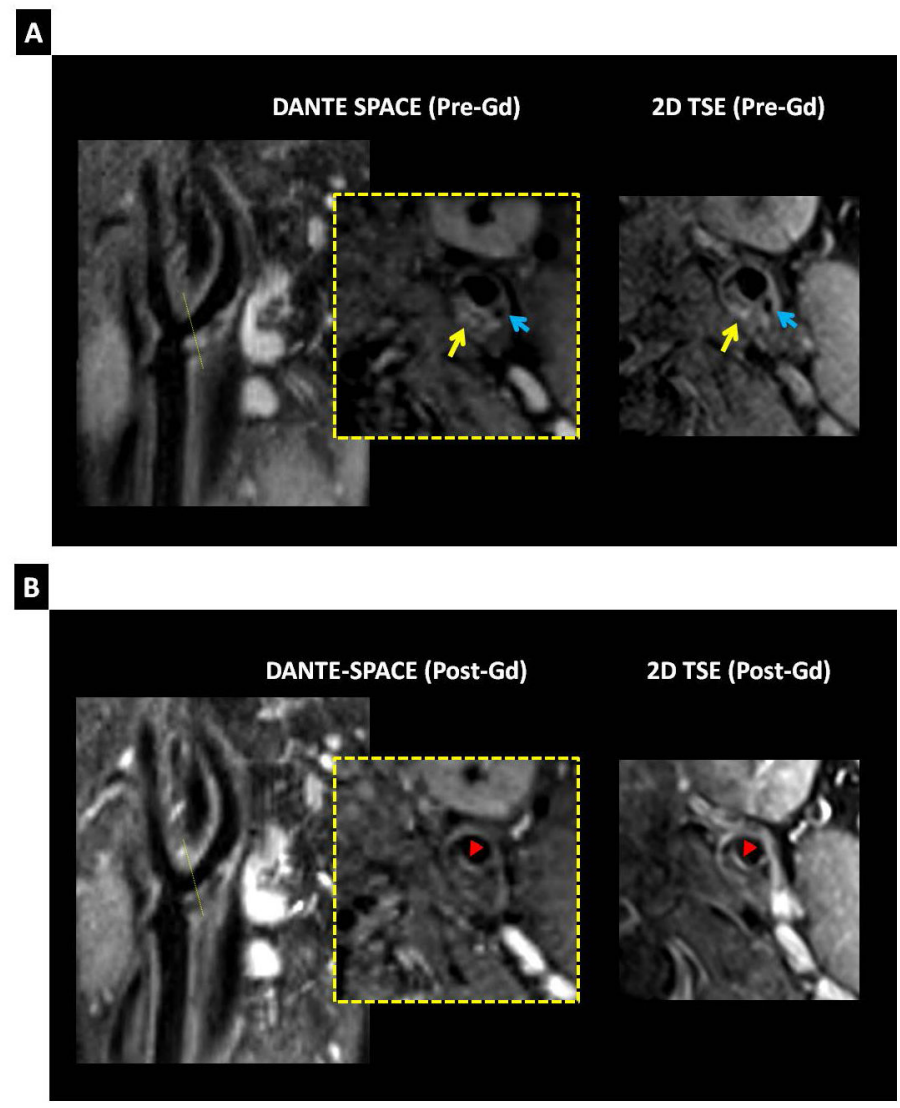

Figure 8.

Reformatted pre- and post-CE DANTE-SPACE images of a vulnerable plaque. The patient was a 41 y/o male with stroke symptoms. Slice-matched T1w 2D TSE images were acquired as reference. (A) Pre-CE DANTE-SPACE images at the bifurcation showed hyperintensive plaque component suggesting intraplaque hemorrhage (yellow arrows). Also note the hypointense plaque component suggesting calcification (blue arrows). (B) Post-CE DANTESPACE images showed enhanced plaque component next to the lumen suggesting thin fibrous cap (red arrows). 


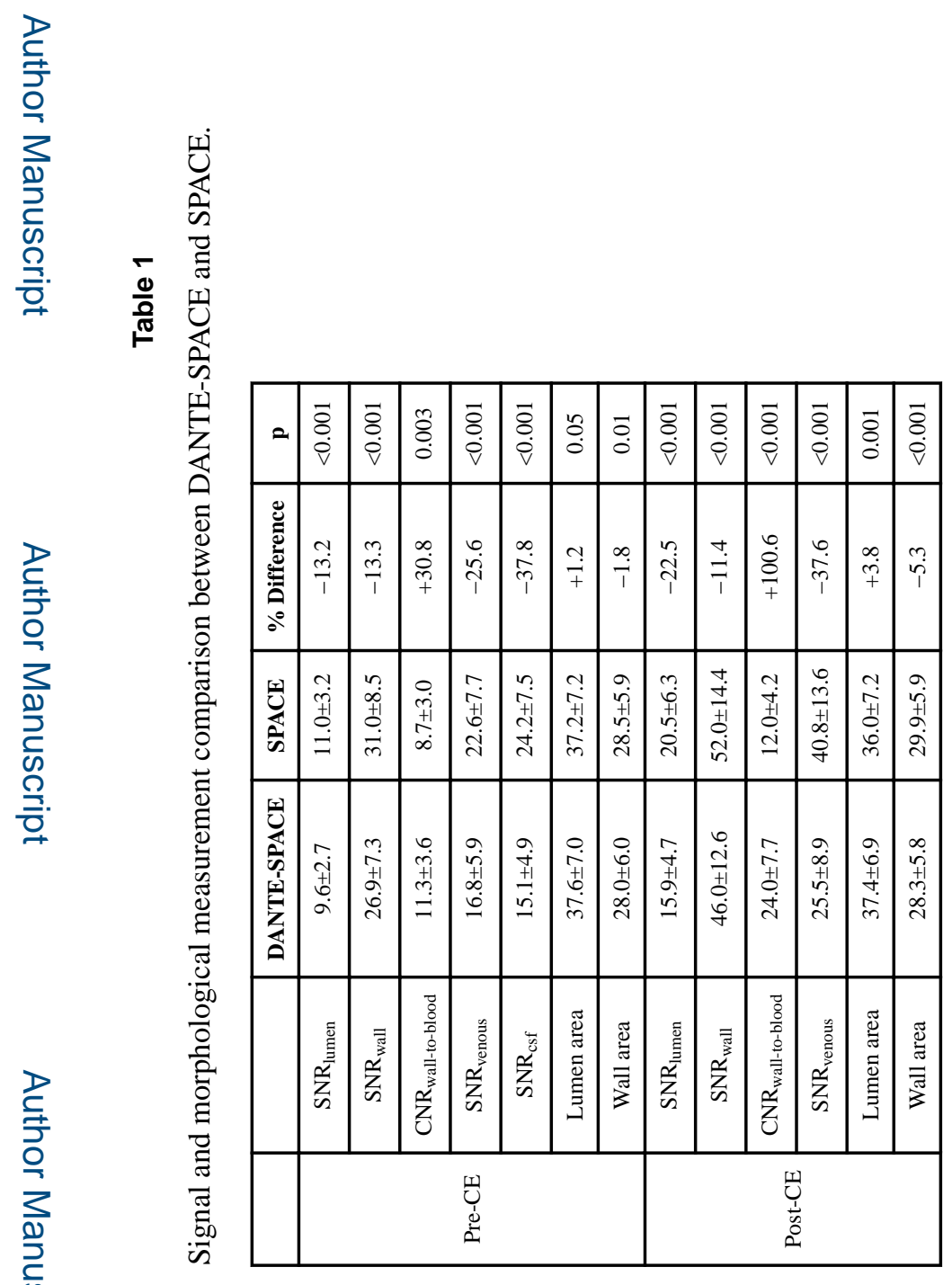

